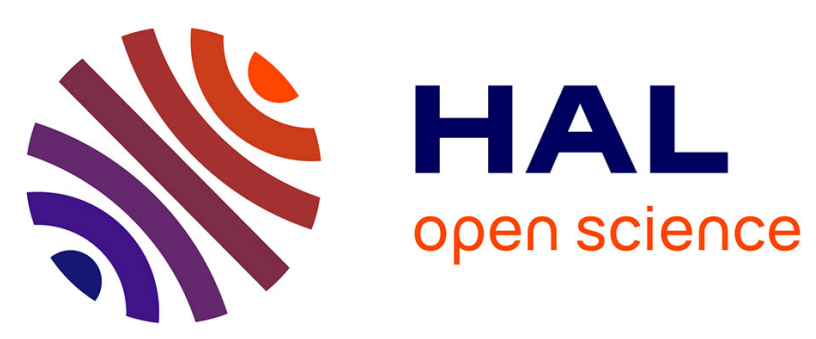

\title{
Thermal noise reduction in interferometric gravitational wave antennas: using high order TEM modes
}

\author{
B. Mours, E. Tournefier, J.-Y. Vinet
}

\section{To cite this version:}

B. Mours, E. Tournefier, J.-Y. Vinet. Thermal noise reduction in interferometric gravitational wave antennas: using high order TEM modes. Classical and Quantum Gravity, 2006, 23, pp.5777-5784. 10.1088/0264-9381/23/20/001 . in2p3-00113125

\section{HAL Id: in2p3-00113125 \\ https://hal.in2p3.fr/in2p3-00113125}

Submitted on 13 Nov 2006

HAL is a multi-disciplinary open access archive for the deposit and dissemination of scientific research documents, whether they are published or not. The documents may come from teaching and research institutions in France or abroad, or from public or private research centers.
L'archive ouverte pluridisciplinaire HAL, est destinée au dépôt et à la diffusion de documents scientifiques de niveau recherche, publiés ou non, émanant des établissements d'enseignement et de recherche français ou étrangers, des laboratoires publics ou privés. 


\title{
Thermal noise reduction in Interferometric Gravitational Wave Antennas: Using high order TEM modes
}

\author{
Benoît Mours, Edwige Tournefier \\ Laboratoire d'Annecy-le-Vieux de Physique des Particules \\ BP 110, 74941 Annecy-le-Vieux Cedex \\ benoit.mours@lapp.in2p3.fr, \\ edwige.tournefier@lapp.in2p3.fr
}

\section{Jean-Yves Vinet}

Dpt. ARTEMIS, Observatoire de la Côte d'Azur, BP 4229, 06304 Nice, France.

vinet@obs-nice.fr

\begin{abstract}
We compute the low frequency tail of the power spectral density of thermal noise in the case of an optical Fabry-Perot resonant cavity operating with Laguerre-Gauss modes of order higher than $(0,0)$. We show a significant reduction of the thermal noise as the order of the mode increases. We discuss the diffraction losses.
\end{abstract}

pacs 04.80.Nn; 05.40.+j

\section{Introduction}

In the central region of the bandwidth of present ground based interferometric gravitational wave (GW) antennas, the main limitation to sensitivity comes from fluctuations of mirrors surfaces under random motions of matter in the bulk material of the substrate. These fluctuations are interpreted by the readout optical beam as a true motion of the mirror, or a GW signal as well. It has been shown [1] that using a "flat-top" mode in the long resonant cavities of GW interferometric detectors results in a large reduction of internal noises of mirrors, due to an averaging process on an area of the mirror's surface larger than with a fundamental gaussian beam. A model for building such modes and the corresponding mirrors has even been developed [2]. The drawback is that one must forget spherical mirrors and produce exotic ("Mexican hat") surfaces.

Operating a Virgo-like optical system with such mirrors raises technological problems presently under investigation. Practical realization of such mirrors seem feasible, but the requirements on angular control must be carefully assessed.

Using Laguerre-Gauss modes of orders larger than $(0,0)$ could have an effect analogous to flat modes by extending the power distribution on the mirror's surface. Even if the achieved noise reduction is lower than with flat modes, it is interesting to keep spherical mirrors and possibly avoid the above mentionned technological issues.

This is why it was interesting to compute the spectral density of noise in such a case and test this conjecture.

We shall see at the end that the reduction factor turns out to be even larger than with flat modes, depending on the orders of the mode. 


\section{The BHV model}

In the case where both the mirrors and the beam are assumed axisymmetrical, there exists a model allowing an accurate calculation of the low frequency tail of the spectral densities of internal noises. As a result of Levin's [3] theory The power spectral density (PSD) of displacement equivalent to thermal noise takes the general (low frequency) form :

$$
S_{x}(f)=\frac{4 k_{B} T}{\pi f} \phi U
$$

where $\phi$ is a loss angle, and where $U$ is the strain energy of the mirror under a pressure distribution having the same profile as the readout beam, and normalized to $1 \mathrm{~N}$.

Then an analytical method for computing $U$ has been developped in [BHV] [4] then amended in [5]. For summarizing the result, the total internal energy is the sum of two contributions:

$$
U=U_{0}+\Delta U
$$

that can be computed separately. Let $a$ be the radius of the mirror and $h$ its thickness. Let $J_{\nu}(x)$ be the Bessel functions, and $\left\{\zeta_{k}, k>0\right\}$ the family of all non-zero solutions of $J_{1}(\zeta)=0$. Let us note $x_{k} \equiv \zeta_{k} h / a$, and $q_{k} \equiv \exp \left(-2 x_{k}\right)$. Let $Y$ be the Young modulus of the mirror's material and $\sigma$ its Poisson ratio.

Then we have:

$$
U_{0}=\frac{1-\sigma^{2}}{\pi a Y} \sum_{k>0} \frac{J_{0}^{2}\left(\zeta_{k}\right) p_{k}^{2}}{\zeta_{k}} \frac{1-q_{k}^{2}+4 q_{k} x_{k}}{\left(1-q_{k}\right)^{2}-4 q_{k} x_{k}^{2}}
$$

The dimension of $U$ is $\mathbf{J} . \mathrm{N}^{-2}$. (See the VPB [6] for details).

In the preceding expression the Fourier-Bessel coefficients $\left\{p_{k}, k>0\right\}$ are determined by the pressure profile. If we denote by $p(r)$ this pressure distribution, we have:

$$
p_{k}=\frac{2 \pi}{J_{0}^{2}\left(\zeta_{k}\right)} \int_{0}^{a} p(r) J_{0}\left(\zeta_{k} r / a\right) r d r
$$

For the second contribution, we have:

$$
\Delta U=\frac{a^{2}}{6 \pi h^{3} Y}\left[\left(\frac{h}{a}\right)^{4}+12 \sigma \xi\left(\frac{h}{a}\right)^{2}+72(1-\sigma) \xi^{2}\right]
$$

with

$$
\xi \equiv \sum_{k>0} p_{k} J_{0}\left(\zeta_{k}\right) / \zeta_{k}^{2}
$$

At this level, the computation amounts to find the $p_{k}$.

\section{Power profiles}

In the case of a Gaussian $\mathrm{TEM}_{00}$ readout mode, we consider its normalized amplitude

$$
\Psi_{0,0}(r)=\sqrt{\frac{2}{\pi w^{2}}} \exp \left(-r^{2} / w^{2}\right)
$$

the pressure distribution is then:

$$
p(r)=\left|\Psi_{0,0}(r)\right|^{2}
$$

and the $p_{k}$ coefficients are [4]:

$$
p_{k, 0}^{0}=\frac{1}{J_{0}\left(\zeta_{k}\right)^{2}} \exp \left[-\frac{\zeta_{k}^{2} w^{2}}{8 a^{2}}\right]
$$

The preceding result can be extended to the case of any axisymmetrical Laguerre-Gauss mode $\mathrm{LG}_{n, m}$. It is well known that the paraxial diffraction equation (relevant for finding the eigenmodes of a resonant cavity with weakly spherical mirrors) admits solutions of the form (in polar coordinates)

$$
\Psi_{n, m}(r, \phi, z)=\sqrt{\frac{2}{\pi w(z)^{2}} \frac{m !}{(m+n) !}} \exp \left(-r^{2} / w(z)^{2}\right)\left(2 r^{2} / w(z)^{2}\right)^{n / 2} L_{m}^{(n)}\left(2 r^{2} / w(z)^{2}\right) \times
$$




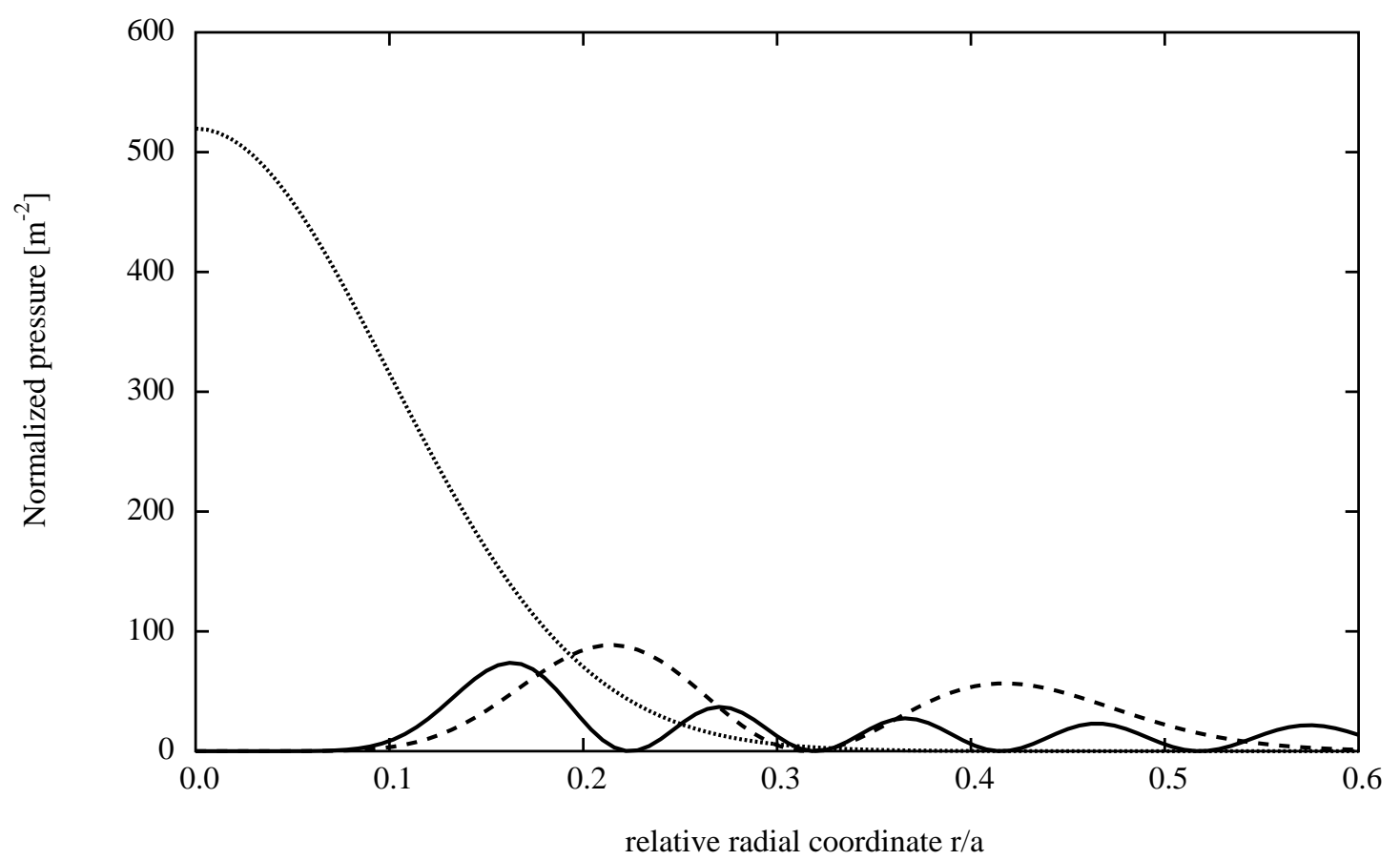

Figure 1. Pressure profiles. $(0,0)$ : dotted, $(4,1)$ : dashed, $(5,5)$ : solid

$$
\times \exp (i n \phi) \exp \left[-i(2 m+n+1) \arctan \left(z / z_{R}\right)\right] \exp \left[i \pi r^{2} / \lambda R(z)\right]
$$

where the $L_{m}^{(n)}(x)$ are the Generalized Laguerre polynomials. $\lambda$ is the wavelength and $z_{R}$ the Rayleigh parameter. The functions $w(z)$ and $R(z)$ define respectively the half-width of the mode, and the curvature radius of its wavefront. In what follows, we only need $w(z)$. At the location $z_{M}$ of a mirror, the normalized pressure distribution has therefore the general expression $\left(w \equiv w\left(z_{M}\right)\right)$ :

$$
p_{m}^{n}(r)=\frac{2}{\pi w^{2}} \frac{m !}{(m+n) !} \exp \left(-2 r^{2} / w^{2}\right)\left(2 r^{2} / w^{2}\right)^{n} L_{m}^{(n)}\left(2 r^{2} / w^{2}\right)^{2}
$$

If the ratio $w / a$ is large enough that the diffraction losses are small, we can replace the finite upper bound of integral (3) by $+\infty$, and the Fourier-Bessel coefficients are simply:

$$
p_{k, m}^{n}=\frac{1}{J_{0}\left(\zeta_{k}\right)^{2}} \exp \left[-\frac{\zeta_{k}^{2} w^{2}}{8 a^{2}}\right] L_{m}^{(0)}\left(\frac{\zeta_{k}^{2} w^{2}}{8 a^{2}}\right) L_{n+m}^{(0)}\left(\frac{\zeta_{k}^{2} w^{2}}{8 a^{2}}\right)
$$

If the diffraction losses are not small, the preceding expression losses some accuracy, but simultaneously the corresponding mode losses its practical interest. The pressure profiles on the input mirror for some among the first Laguerre-Gauss modes of parameter $w=3.5 \mathrm{~cm}$ are represented on Fig.1. See on Fig.?? the intensity pattern of a $\mathrm{LG}_{5,5}$ (for instance) mode. The integrated power (Fig.3) clearly show a smoother distribution of power on the mirror's surface. The virtual deformation of the mirror's surface under a pressure normalized to $1 \mathrm{~N}$ can be seen on Fig. 4 with again $w=3.5 \mathrm{~cm}$. The radius of the mirror is assumed $a=17.5 \mathrm{~cm}$, its thickness $h=10 \mathrm{~cm}$. One clearly sees that the strain is a decreasing function of the orders $((n, m))$ of the mode.

\section{Relative gains on thermal noise}

With the current parameters $a=17.5 \mathrm{~cm}, h=10 \mathrm{~cm}, w=2 \mathrm{~cm}$ (For instance Virgo input mirrors), if we insert the preceding $p_{k, m}^{n}$ in equations 2 and 4 , we find for the fundamental:

$$
U_{0}^{(00)}=1.8110^{-10} \mathrm{~J} . \mathrm{N}^{-2} \Delta U^{(00)}=210^{-11}{\mathrm{~J} . \mathrm{N}^{-2}}^{-2} U^{(00)}=2.0210^{-10}{\mathrm{~J} . \mathrm{N}^{-2}}^{-2}
$$




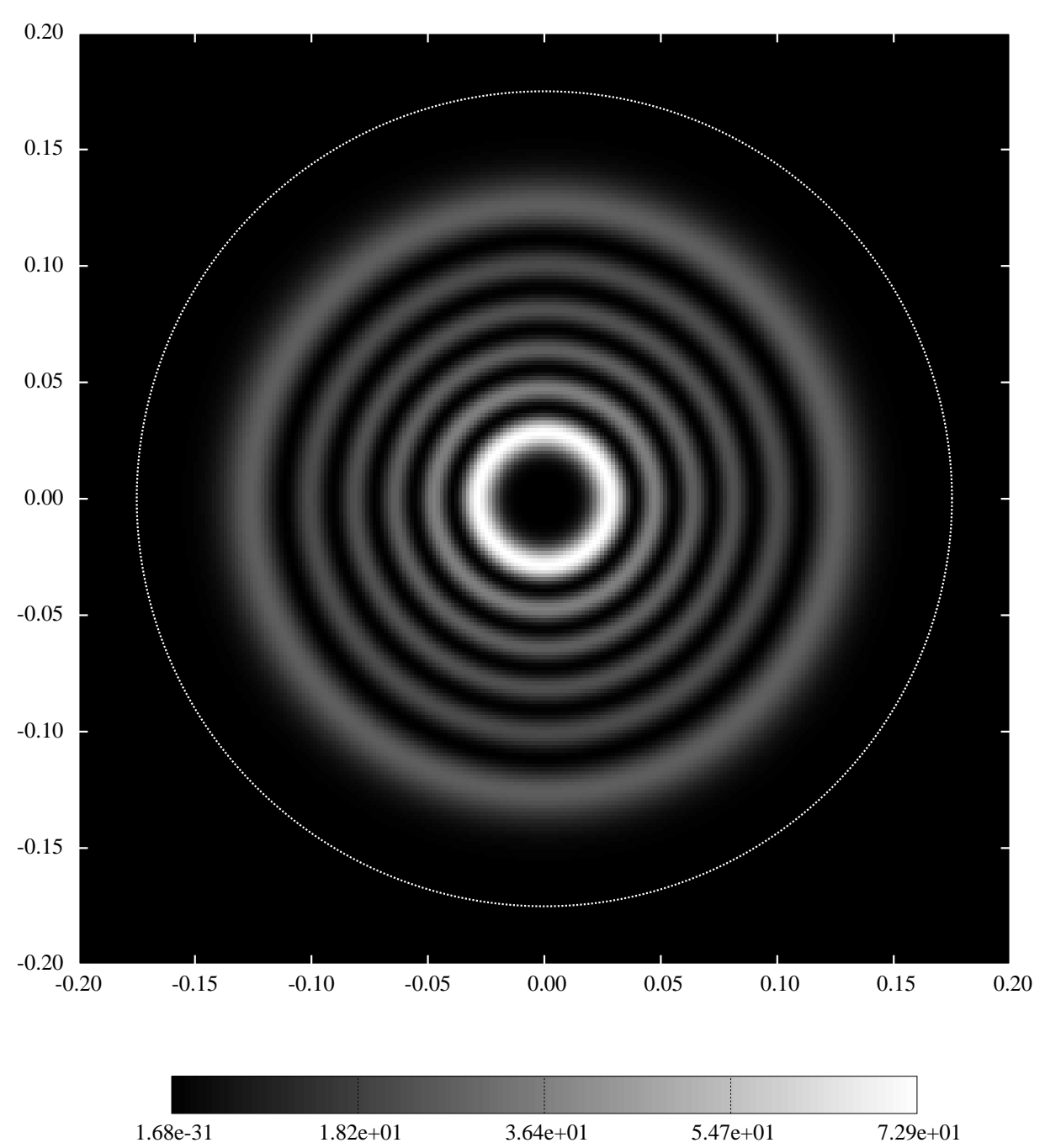

Figure 2. Intensity pattern of a $\mathrm{LG}_{5,5}$ mode of Gaussian parameter $w=3.5 \mathrm{~cm}$. The faint circle represents the edge of a $17.5 \mathrm{~cm}$ radius mirror (units: $\mathrm{m}$ ).

then, for instance

$$
U_{0}^{(01)}=1.0310^{-10} \mathrm{~J} . \mathrm{N}^{-2} \Delta U^{(01)}=210^{-11}{\mathrm{~J} . \mathrm{N}^{-2}}^{-2} U^{(01)}=1.2310^{-10}{\mathrm{~J} . \mathrm{N}^{-2}}^{-2}
$$

and

$$
\begin{aligned}
& U_{0}^{(02)}=7.6310^{-11} \mathbf{J} . \mathrm{N}^{-2} \Delta U^{(02)}=1.910^{-11} \mathbf{J} . \mathrm{N}^{-2} \Rightarrow U^{(02)}=9.5110^{-11} \mathbf{J} . \mathrm{N}^{-2} \\
& U_{0}^{(03)}=6.1310^{-11} \mathbf{J} . \mathrm{N}^{-2} \Delta U^{(03)}=1.7810^{-11} \mathbf{J} . \mathrm{N}^{-2} \Rightarrow U^{(03)}=7.9110^{-11} \mathbf{J} . \mathrm{N}^{-2}
\end{aligned}
$$

For comparison, with a flat mode of radius $0.1 \mathrm{~m}$, we have

$$
U_{0}^{\text {flat }}=1.6010^{-11} \mathrm{~J} . \mathrm{N}^{-2} \Delta U^{\text {flat }}=1.110^{-11} \mathrm{~J} . \mathrm{N}^{-2} \Rightarrow U^{\text {flat }}=2.6510^{-11} \mathrm{~J} . \mathrm{N}^{-2}
$$




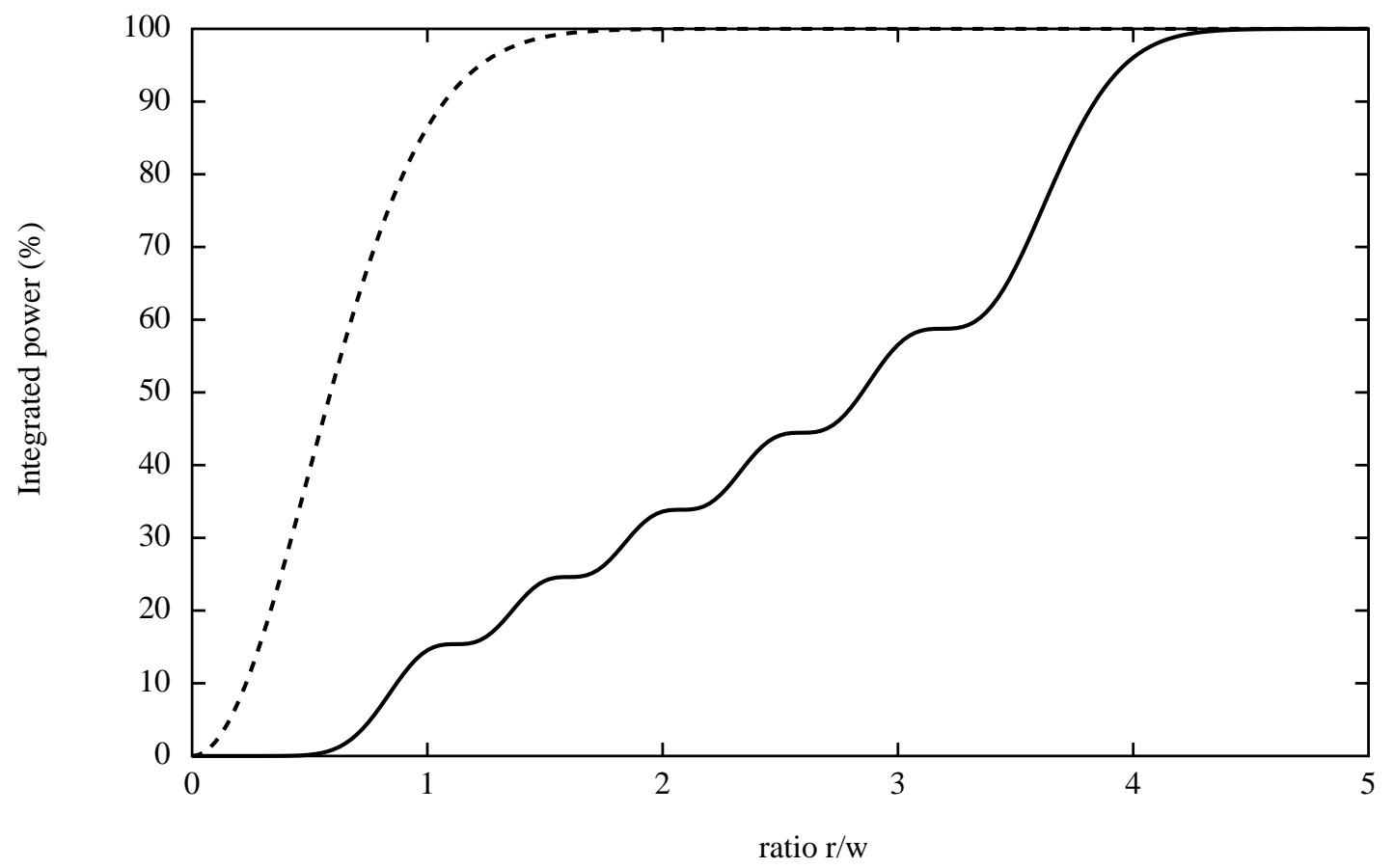

Figure 3. Integrated power for $\mathrm{LG}_{5,5}$ (solid line) and $\mathrm{LG}_{0,0}$ (dashed line) $(w=3.5 \mathrm{~cm})$
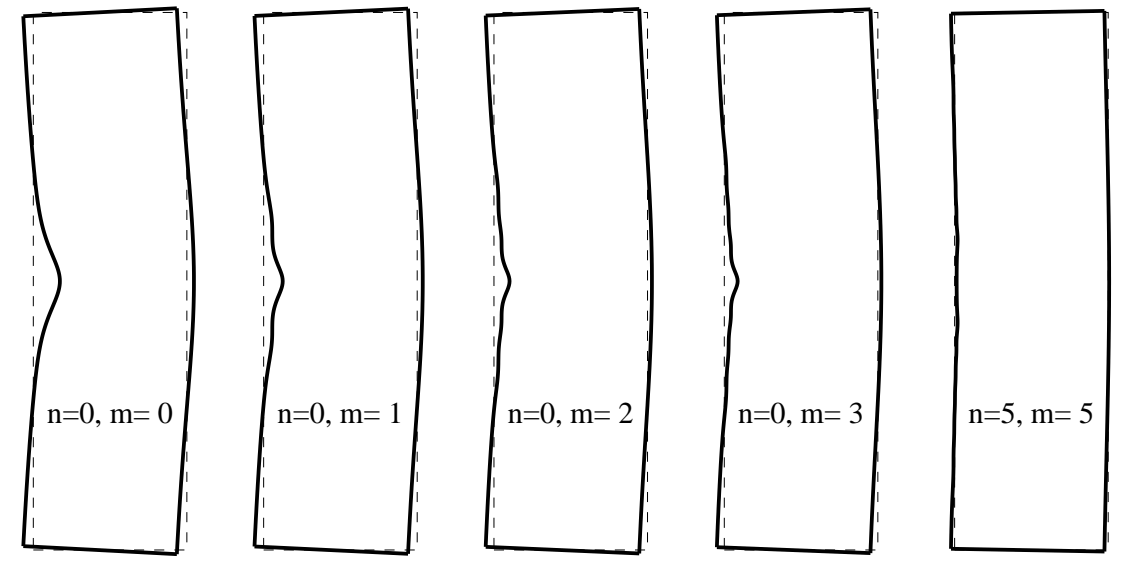

Figure 4. Deformation under $\mathrm{LG}_{n, m}$ readout beam $(w=3.5 \mathrm{~cm})$ of a cylindrical mirror $(a=17.5 \mathrm{~cm}, h=10$ $\mathrm{cm})$. (exaggerated by a factor of $6 \times 10^{7}$ ) 


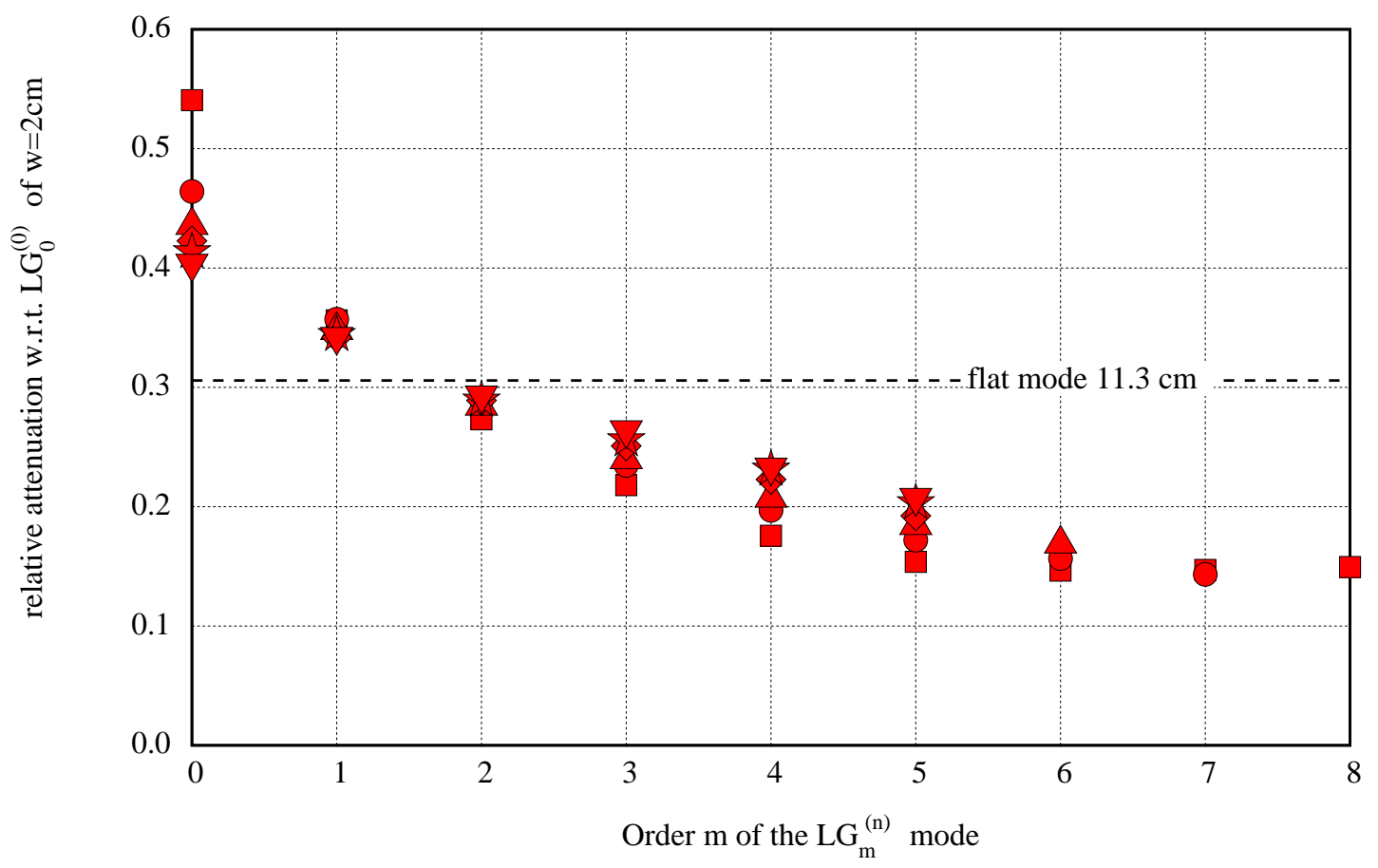

Figure 5. Relative reduction in root spectral density of thermal noise vs order $m$ of the LG mode (each 1 ppm diffraction losses). $n=0, \ldots, 8$ (from squares to down pointing triangles)

The gains in root spectral density of noise are for the first modes:

$$
\begin{aligned}
& \sqrt{U^{(01)} / U^{(00)}} \sim 0.78 \\
& \sqrt{U^{(02)} / U^{(00)}} \sim 0.69 \\
& \sqrt{U^{(03)} / U^{(00)}} \sim 0.63 \\
& \sqrt{U^{\text {flat } / U^{(00)}}} \sim 0.36
\end{aligned}
$$

For comparison, we consider a flat mode as proposed in [2], built by convolution of an elementary TEM $_{0,0}$ of half-width $w_{0}$ with a disk of radius $b$. For a 17.5 radius mirror, the parameters could be $w=3 \mathrm{~cm}, b=$ $11.3 \mathrm{~cm}$. By increasing the order of the LG mode and the beam half-width $w$, it is possible to reach gains comparable to this flat mode. On Fig.5, we show the gains relative to the standard situation of Virgo $\left(\mathrm{LG}_{(0,0)}\right.$, $\mathrm{w}=2 \mathrm{~cm}$ ) for several higher order modes having each a $w$ parameter adjusted to set diffraction losses at $1 \mathrm{ppm}$; the gain of a flat mode of size $11.3 \mathrm{~cm}$ (having the same losses is also shown). On Fig.6 we show the ratio $a / w$ insuring diffraction losses of 1 ppm, versus orders of the mode. Unfortunately, the diffraction losses also increase with the mode order. They remain negligible on the input mirror, where the Gaussian parameter of the beam is $w_{0}=2 \mathrm{~cm}$, but they explode on the end mirror where the beam parameter is $w_{L}=5.46 \mathrm{~cm}$. This is a consequence of the design of the Virgo cavity involving a flat input mirror and a spherical end mirror. Symmetrical cavities with two spherical identical mirrors are also possible. In this case, beam sizes about 3 cm give acceptable results (see Fig..7).

To be specific, let us compute the spectral densities of displacement equivalent to thermal noise in three cases. Firstly, in the case of a $\operatorname{TEM}_{0,0}, w=2 \mathrm{~cm}$ beam on a Virgo input mirror:

$$
S_{x}^{1 / 2}(f)=1.0310^{-19}\left[\frac{100 \mathrm{~Hz}}{f}\right]^{1 / 2} \mathrm{~m} \cdot \mathrm{Hz}^{-1 / 2}
$$




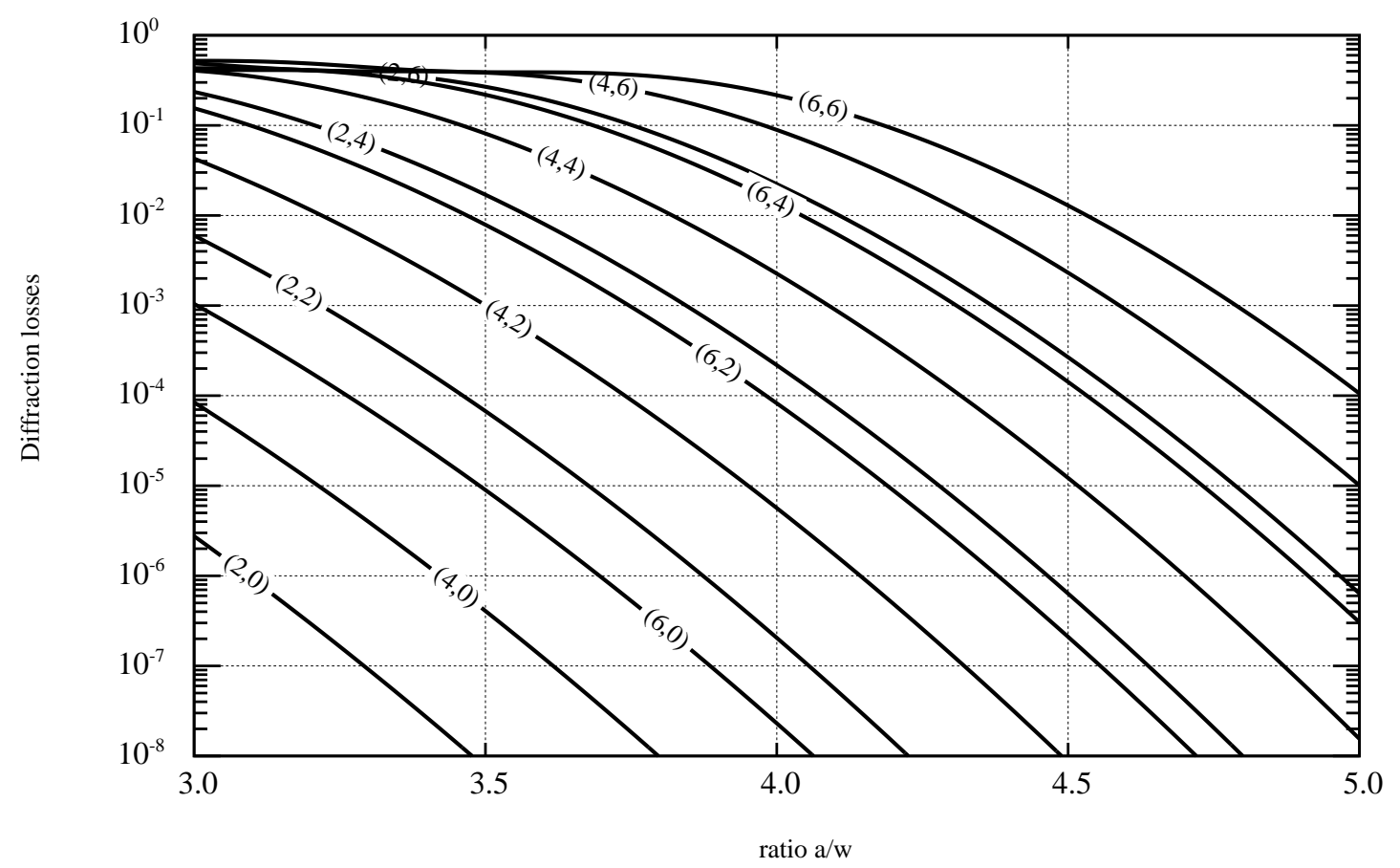

Figure 6. Diffraction losses of $\mathrm{LG}_{n, m}$ modes vs ratio a/w

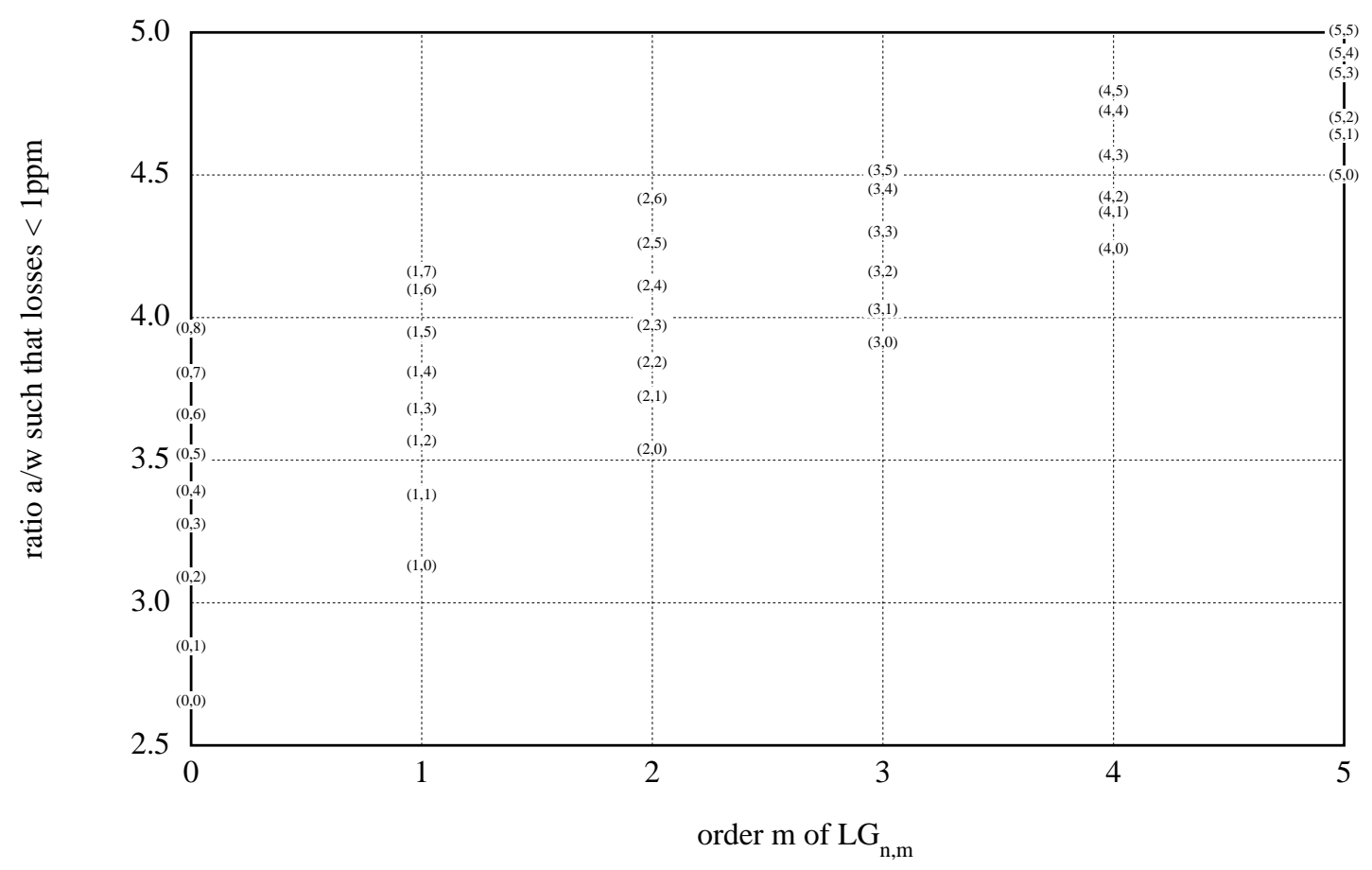

Figure 7. ratio a/w insuring $1 \mathrm{ppm}$ diffraction losses of vs order of the LG mode 
then for a $w=3.5 \mathrm{~cm}$ :

$$
S_{x}^{1 / 2}(f)=7.9010^{-20}\left[\frac{100 \mathrm{~Hz}}{f}\right]^{1 / 2} \mathrm{~m} \cdot \mathrm{Hz}^{-1 / 2}
$$

and now for a $\mathrm{LG}_{5,5}, w=3.5 \mathrm{~cm}$ :

$$
S_{x}^{1 / 2}(f)=1.6910^{-20}\left[\frac{100 \mathrm{~Hz}}{f}\right]^{1 / 2} \mathrm{~m} \cdot \mathrm{Hz}^{-1 / 2}
$$

If we compare the 1st example (standard Virgo) to the third (in some "advanced Virgo"), we note a gain factor of $\sim 6$ in sensitivity in the $100 \mathrm{~Hz}$ region.

\section{Conclusion and perspectives}

It is possible to have a reduction of thermal noise comparable to or even better than that obtained with flat modes, by using moderately high order (roughly $n+m \geq 6$ ) Laguerre-Gauss axisymmetrical modes. It seems beneficial that these modes are compatible with spherical cavity mirrors instead of "Mexican" surface shapes. The diffraction losses on the end mirror would however be too large in the present design (flat/spherical) of the Virgo cavities (2 cm waist on flat input mirror). If the flat/spherical cavities are replaced by symmetrical spherical/spherical cavities, this issue could be overcome. The question of generation of such modes having complex annular patterns could be solved by the recently developed fiber technology (Bragg fibers), allowing to design fiber-lasers with comparable mode structures. A study of the optical stability of such a cavity operating with a $\mathrm{LG}_{n, m}$ mode is now necessary to confirm that it could be practically operated this way. In particular, the issue of the degeneracy of modes having the same $n+2 m$ is to be addressed. 


\section{[1] J-Y. Vinet}

Class. Quant. Grav. 22 (2005) P.1395

[2] E. D'Ambrosio and K. S. Thorne

Phys. Rev. D 67102004

[3] Yu. Levin

Phys. Rev. D 57 (1998) p.659

[4] F. Bondu, P. Hello, J-Y. Vinet

Phys. Lett. A 246 (1998) p.227

[5] Yuk Tung Liu, Kip S. Thorne

Phys. Rev. D 62, 122002

[6] The Virgo Physics Book

http://wwwcascina.virgo.infn.it/vpb/ 